\title{
Supporting Information: \\ The sequencing and interpretation of the genome obtained from a Serbian individual
}

\author{
Wazim Mohammed Ismail ${ }^{1 *}$, Kymberleigh A. Pagel ${ }^{1 *}$, Vikas Pejaver ${ }^{1}$, Simo \\ V. Zhang ${ }^{1}$, Sofia Casasa ${ }^{2}$, Matthew Mort ${ }^{3}$, David N. Cooper ${ }^{3}$, Matthew W. \\ $\mathrm{Hahn}^{1,2}$, and Predrag Radivojac ${ }^{4}$ \\ ${ }^{1}$ Department of Computer Science, Indiana University, Bloomington, Indiana, U.S.A. \\ ${ }^{2}$ Department of Biology, Indiana University, Bloomington, Indiana, U.S.A. \\ ${ }^{3}$ Institute of Medical Genetics, Cardiff University, Cardiff, U.K. \\ ${ }^{4}$ College of Computer and Information Science, Northeastern University, Boston, Massachusetts, U.S.A.
}

\section{Read depth}

The genome sequencing and mapping achieved an average read depth of 34.7, with $98.3 \%$ of GRCh37 reference bases having coverage of 10 -fold or more and $89.4 \%$ having coverage of 20 -fold or more. When only the non-zero positions were considered, the average read depth was 34.8, with $98.5 \%$ of GRCh37 reference bases having coverage of 10-fold or more and $89.7 \%$ having coverage of 20 -fold or more. The number of zero-depth positions were $7,649,443(0.3 \%)$. The depth of coverage distribution obtained using the two read mappers BWA and Bowtie2, before and after read processing - duplicate removal, base quality score recalibration and indel realignment, is shown in Supplementary Figure 1.

Figure 1: Histogram of depth of coverage obtained by two read mappers BWA and Bowtie2 before and after read processing - duplicate removal, base quality score recalibration and indel realignment.

\section{Characterization of variant properties}

Figure 2: Read depth across SNV and indel variants called by the four pipelines.

Figure 3: Size comparison of insertion and deletion variants across the four pipelines.

\footnotetext{
${ }^{*}$ Contributed equally to this work.
} 
Table A: The total number of variants identified by four read mapper vs. variant caller platforms, collected from Figure 1 in the main paper. The results show significant variability of Platypus across two read mappers and also significant variability of Bowtie 2 across two variant callers. We therefore selected a combination of BWA-MEM and GATK as our main platform for single nucleotide and short indel variants.

\begin{tabular}{|c|c|c|}
\hline & Bowtie2 & BWA-MEM \\
\hline Platypus & $2,850,611$ & $3,381,575$ \\
\hline GATK & $3.248,823$ & $3,280,675$ \\
\hline
\end{tabular}

Table B: Number of structural variants called by individual SV callers in SVE

\begin{tabular}{|l|r|r|r|r|r|r|}
\hline SV Caller & Deletion & Duplication & Insertion & Inversion & Translocation & Breakend \\
\hline BreakDancer & 8489 & 903 & 7028 & 451 & 208 & 0 \\
\hline BreakSeq2 & 1993 & 0 & 140 & 0 & 0 & 0 \\
\hline cnMOPS & 3931 & 257 & 0 & 0 & 0 & 0 \\
\hline CNVnator & 10577 & 832 & 0 & 0 & 0 & 0 \\
\hline DELLY & 12561 & 6969 & 943995 & 5469 & 62812 & 0 \\
\hline Hydra & 5159 & 1337 & 0 & 76 & 0 & 176902 \\
\hline LUMPY & 5759 & 550 & 0 & 61 & 0 & 2692 \\
\hline FusorSV & 848 & 3 & 0 & 0 & 0 & 0 \\
\hline
\end{tabular}

Figure 4: Number of heterozygous and homozygous SNV and indel variants called by the four pipelines.

Figure 5: Size comparison of indel variants in the Serbian genome.

\section{Estimate of novel variation from the first individual of a newly sequenced population}

Figure 6: The amount of novel variation observed in each 1000 Genomes Project participant compared to participants from other populations. The peak on the right side is comprised of individuals of African ancestry.

\section{Length distribution of deletions called by FusorSV}

Figure 7: Length distribution of deletions called by FusorSV. 
Table C: Genes that overlap with deletions called by FusorSV.

\begin{tabular}{|c|c|c|c|c|c|c|}
\hline A2ML1 & $A B C B 5$ & $A B I 3 B P$ & $A C A C A$ & $A C O T 11$ & ACSL3 & $A D A M 12$ \\
\hline$A D C Y 8$ & $A H N A K 2$ & $A K A P 6$ & AKT3 & AL162389.1 & $A M D H D 1$ & $A N K R D 30 B L$ \\
\hline$A N K R D 55$ & $A P 3 S 1$ & ARHGAP19 & ARHGAP19-SLIT1 & $A S I P$ & $A S M T$ & ATP10A \\
\hline$A T P 9 B$ & AVL9 & $B A N K 1$ & $B C A R 3$ & $B C A S 3$ & $B C L A F 1$ & $B O P 1$ \\
\hline$B P G M$ & C10orf11 & C11orf49 & C15orf57 & C17orf 85 & C2CD2 & C2orf73 \\
\hline C8orf12 & $C A C N A 1 B$ & $C A C N B 2$ & $C A S P 8$ & $C A S T$ & $C A V 2$ & CCSER 1 \\
\hline$C D 2 A P$ & $C D C 40$ & $C D H 1$ & CDK19 & CEP72 & CHST9 & CLASP2 \\
\hline $\mathrm{CLIC}_{4}$ & $C M S S 1$ & CNOT10 & CNTNAP2 & CNTNAP4 & COL23A1 & COL5A2 \\
\hline$C S M D 1$ & $C T D-2054 N 24.2$ & $C T D-3105 H 18.18$ & CTNNA3 & $C Y P 2 D 6$ & CYP4F3 & $D 2 H G D H$ \\
\hline$D A B 1$ & DACH2 & DACT2 & $D C C$ & DENND1B & $D I P 2 B$ & $D K K 2$ \\
\hline$D L E U 1$ & $D M P K$ & DNAJC27 & DOCK5 & DOCK 8 & DPF3 & $\mathrm{DPH} 7$ \\
\hline$D P P 6$ & $D Y M$ & $E D A R A D D$ & $E G F R$ & EGLN3 & EPHB1 & EPSTI1 \\
\hline$E R C 1$ & ERICH1 & ESR1 & EXOC2 & $E Y S$ & FAM149A & $F A M 154 B$ \\
\hline FAM160A1 & FAM203B & FAM20C & FВХОЗ6 & FHIT & FILIP1L & FKBP5 \\
\hline$F M N 2$ & FRMD5 & $G A B R G 1$ & GALNT18 & GALNTL6 & $G B P 3$ & $G D A P 1$ \\
\hline GNG2 & GNGT1 & GPR156 & GRIK3 & GRIN3A & $H A C E 1$ & HCN2 \\
\hline HHAT & $H I G D 1 A$ & $H L A-A$ & $H S F 2 B P$ & IFT52 & $I G H D 1-20$ & $I G H D 2-21$ \\
\hline IGHD6-19 & $I G H V 4-61$ & $I L 2 R A$ & $I Q C J-S C H I P 1$ & $I Q G A P 3$ & $K C N A B 1$ & KCNIP1 \\
\hline KCNJ12 & КIAА0391 & KIAA1217 & KLHL3 & $K L R C 1$ & $K L R C 2$ & $K M-P A-2$ \\
\hline KRT77 & KRTAP9-6 & KRTAP9-7 & LARS2 & $L C E 3 B$ & $L C E 3 C$ & $L E P R E L 1$ \\
\hline$L G I 1$ & $L H F P$ & LITAF & $L R R N 4$ & $L T B P 1$ & LUZP2 & MACROD2 \\
\hline$M A G T 1$ & MAPK10 & MATR3 & METAP1D & METTL21A & METTL24 & $M G A M$ \\
\hline$M G A T 5$ & MID1 & MITF & $M L I P$ & MROH1 & $M R P L 42$ & MSI2 \\
\hline MSR1 & MUC3A & $M Y C T 1$ & MYO5B & MYO9A & $N A L C N$ & $N B E A L 1$ \\
\hline NCOA7 & $N E L L 1$ & $N K G 2-E$ & $N R G 3$ & $N R X N 3$ & NT5M & OIP5 \\
\hline$O_{4} N_{4}$ & OR52N1 & OR52N5 & $P_{A D I}$ & PAPРA2 & PARD $3 B$ & PARK2 \\
\hline$P C$ & $P C C B$ & PCDH15 & PCDHB10 & PCDHB16 & $P C D P 1$ & PCM1 \\
\hline PCSK5 & $P D E 4 D I P$ & РDLIMЗ & $P D S S 1$ & $P D X K$ & PEX5L & PHYHD1 \\
\hline PIAS1 & PKHD1 & PLEKHA & POU6F2 & $P P A P D C 1 A$ & PPFIA2 & $P P M 1 B$ \\
\hline$P P P 1 R 1 C$ & PPP1R3A & PPP2R3B & PPP2R 5A & $P P P 4 R 2$ & PRIM2 & PRPSAP2 \\
\hline PSD3 & PTPRD & PTPRK & PTPRN2 & PTPRT & $P W P 2$ & $R A D 51 B$ \\
\hline$R A L Y L$ & $R A P 1 G A P 2$ & $R A S G E F 1 B$ & $R B P J$ & RFPL4A & RIMS1 & $R N F 144 B$ \\
\hline RNF212 & RNF38 & RP11-192H23.4 & RP11-215A19.2 & RP4-576H24.4 & RРНЗАL & RPIA \\
\hline RPTOR & RTDR1 & $S 1 P R 4$ & $S A A L 1$ & SBF2 & $S C A P$ & SCAPER \\
\hline$S C M L 4$ & $S C N N 1 D$ & $S C X A$ & $S C X B$ & SERAC1 & SERPINA1 & $S G C D$ \\
\hline$S G K 1$ & SGSM3 & SHISA9 & SIRPB1 & $S L C 12 A 8$ & $S L C 1 A 7$ & SLC25A18 \\
\hline SLC35E4 & $S L C 4 A 10$ & SMYD3 & SPAG16 & SPOCK 1 & SPOCK3 & $S P R N$ \\
\hline STEAP2 & STON1 & STON1-GTF2A1L & SUCLG2 & SUMF1 & $S Y T 11$ & $T B C 1 D 16$ \\
\hline$T B C E$ & $T C E R G 1 L$ & $T D G$ & THSD4 & TJP2 & TMBIM4 & TMEM132D \\
\hline TMEM192 & TMEM41B & TOX2 & $\mathrm{TOX}_{4}$ & TPST1 & TRAF3IP3 & TRIM5 \\
\hline TSPAN8 & TUBGCP2 & UGT1A10 & UGT1A4 & UGT1A5 & UGT1A6 & UGT1A7 \\
\hline$U G T 1 A 8$ & UGT1A9 & UGT2B17 & UIMC1 & UNC13B & $U N K L$ & $V M P 1$ \\
\hline WDR34 & $W D R 47$ & $W N K 1$ & $W W O X$ & $W W T R 1$ & XKR 9 & $X R N 2$ \\
\hline $\begin{array}{l}Z \text { Z } 3 H 11 A \\
\text { ZNF665 }\end{array}$ & $\begin{array}{l}Z D H H C 19 \\
Z P B P\end{array}$ & ZMAT4 & ZNF100 & $Z N F 124$ & $Z N F 14$ & ZNF385D \\
\hline
\end{tabular}

Table D: Genes that overlap with duplications called by FusorSV.

\begin{tabular}{|lllll|}
\hline IGHD1OR15-1A & IGHD2OR15-2A & IGHD3OR15-3A & IGHD4OR15-4A & IGHD5OR15-5A \\
IL9R & PPP2R3B & SHOX & SPRY3 & VAMP \\
\hline
\end{tabular}




\section{$5 \quad$ Variants in genotype-phenotype annotation databases}

\subsection{HGMD annotations}

There are five different classes of variant listed in HGMD. Disease-causing mutations (DM) are entered into HGMD where the authors of the corresponding report(s) have established that the reported mutation(s) are involved (or very likely to be involved) in conferring the associated clinical phenotype upon the individuals concerned. The DM classification may, however, also appear with a question mark (DM?), denoting a probable/possible pathological mutation, reported as likely to be disease causing in the corresponding report, but where (i) the author has indicated that there may be some degree of doubt or uncertainty; (ii) the HGMD curators believe greater interpretational caution is warranted, or (iii) subsequent evidence has appeared in the literature which has called the initial putatively deleterious nature of the variant into question (e.g. a negative functional, case-control or populationscale sequencing study). In addition, three categories of polymorphism are included in the database. Disease-associated polymorphisms (DP) are entered into HGMD where there is evidence for a significant association with a disease/clinical phenotype along with additional evidence that the polymorphism is itself likely to be of functional relevance (e.g., as a consequence of genic location, evolutionary conservation, transcription factor binding potential, etc.), although there may be no direct evidence (e.g., from an expression study) for a functional effect. The functional polymorphisms (FP) class includes those sequence changes for which a direct functional effect has been demonstrated (e.g., by means of an in vitro reporter gene assay or alternatively by protein structure, function or expression studies), but with no disease association reported as yet. Disease-associated polymorphisms with supporting functional evidence (DFP) must meet both of the above criteria in that the polymorphism should not only have been reported to be significantly associated with disease, but should also display direct evidence of being of functional relevance.

\subsection{ClinVar annotations}

Several variants within the Serbian genome are characterized with pathogenic Clinical Significance in the ClinVar database. Nine variants are observed in the homozygous state in the Serbian genome which correspond to "Pathogenic" Clinical significance in ClinVar. Of these, seven have been submitted without assertion criteria and represent less confident annotation. The remaining two entries are provided by a single submitter and are similarly not of sufficient confidence to be included explicitly in the manuscript.

There are 25 heterozygous variants annotated as "Pathogenic" in the Serbian genome. Of these, many are low confidence: 11 are submitted without assertion criteria, 4 have criteria provided by a single submitter, and 3 have conflicting interpretations. The remaining 7 variants have criteria provided by multiple submitters without conflicting interpretations. The heterozygous variants are associated with multiple phenotypes, including trimethylaminuria, irido-corneo-trabecular dysgenesis, glaucoma, anterior segment dysgenesis, CYP1B1-related disorders, progressive familial heart block, skin and nail abnormalities, epidermolysis bullosa, butyrylcholine esterase deficiency, postanesthetic apnea, hereditary cancer-predisposing syndromes, and Niemann-Pick disease. Of these phenotypes, none are known to have occurred 
Table E: Frameshifting insertion/deletion variants scored with MutPred-LOF

\begin{tabular}{|l|l|l|l|}
\hline Gene & Variant & Score & Affected Molecular Mechanisms \\
\hline NM_030665 & Q280Hfs*84 & 0.57394 & $\begin{array}{l}\text { Methylation(p=0.003); Amidation(p=0.004); Ubiquity- } \\
\text { lation(p=0.004); MoRF(p=0.005); O-linked glycosyla- } \\
\text { tion(p=0.007) }\end{array}$ \\
\hline NM_030665 & Q280Afs*108 & 0.57334 & $\begin{array}{l}\text { Methylation(p=0.003); Amidation(p=0.004); Ubiquity- } \\
\text { lation(p=0.004); MoRF(p=0.005); O-linked glycosyla- } \\
\text { tion(p=0.007) }\end{array}$ \\
\hline NM_024675 & R170Ifs*14 & 0.54289 & $\begin{array}{l}\text { MoRF(p=0.03); Pyrrolidone carboxylic acid(p=0.033); } \\
\text { Loop(p=0.034); Amidation(p=0.036); O-linked glycosyla- } \\
\text { tion(p=0.038) }\end{array}$ \\
\hline NM_006580 & A56Lfs*16 & 0.52228 & Palmitoylation(p=0.02) \\
\hline NM_001037333 & Q95Pfs*15 & 0.511 & Helix(p=0.015); PPI hotspot(p=0.038) \\
\hline NM_001035235 & V110Dfs*2 & 0.50882 & NA \\
\hline NM_001009931 & M1Cfs*28 & 0.50536 & $\begin{array}{l}\text { Signal cleavage(p=0); RNA binding(p=0); Sulfation(p=0); } \\
\text { B factor(p=0.0001); Copper binding(p=0.0002) }\end{array}$ \\
\hline
\end{tabular}

within the immediate family history.

Table H: Top scoring noncoding variants from CADD

\begin{tabular}{|l|l|l|l|l|}
\hline Chr & Pos & Ref/Alt & Consequence & Score \\
\hline 17 & 59667953 & G/A & noncoding_change & 37 \\
\hline 16 & 88780634 & AGGTGTG/A & downstream & 35 \\
\hline 2 & 85570766 & CAGAT/C & upstream & 35 \\
\hline 17 & 40270029 & $\begin{array}{l}\text { ACTGAAGCTGAGGAGAGAGAGAGACGT } \\
\text { CAGGGATGGGGGG/A }\end{array}$ & downstream & 27 \\
\hline 8 & 145617534 & TGGGGGTGCAAGGTGA/T & downstream & 25.3 \\
\hline 17 & 59668021 & G/C & noncoding_change & 24.4 \\
\hline 22 & 37465385 & CTGGGG/C & regulatory & 24.4 \\
\hline 14 & 77648237 & TGTG/T & regulatory & 24.3 \\
\hline 2 & 61389737 & T/G & noncoding_change & 23.8 \\
\hline 19 & 44610843 & C/T & noncoding_change & 23.8 \\
\hline 6 & 160560897 & CTGGTAAGT/C & regulatory & 23.5 \\
\hline 2 & 20448521 & C/T & upstream & 23.5 \\
\hline 3 & 10368577 & C/A & upstream & 23.3 \\
\hline 12 & 104341644 & AACTG/A & downstream & 23.2 \\
\hline 3 & 6513628 & CACAG/C & intergenic & 23.2 \\
\hline 3 & 96336236 & G/T & upstream & 23.2 \\
\hline 9 & 113128561 & TGAGGGTAG/T & downstream & 23.1 \\
\hline 15 & 37172064 & C/A & downstream & 23.1 \\
\hline 4 & 8785222 & C/G & intergenic & 23.1 \\
\hline 7 & 11874349 & TA/T & upstream & 23.1 \\
\hline 17 & 36782034 & TAAA/T & upstream & 23.1 \\
\hline 21 & 35795163 & T/A & upstream & 23.1 \\
\hline 4 & 24516813 & TTTATC/T & downstream & 23 \\
\hline 7 & 131185995 & C/A & downstream & 23 \\
\hline 10 & 103002063 & G/A & downstream & 23 \\
\hline 11 & 61165731 & C/CA & downstream & 23 \\
\hline 15 & 38982825 & C/CT & downstream & 23 \\
\hline & & & & \\
\hline
\end{tabular}




\begin{tabular}{|c|c|c|c|c|}
\hline 15 & 100514418 & $\mathrm{C} / \mathrm{G}$ & downstream & 23 \\
\hline 17 & 5286861 & GTAGTGTTTGGAATTTTCTGTTCATA/G & downstream & 23 \\
\hline 1 & 91621787 & TAACA/T & intergenic & 23 \\
\hline 4 & 156503230 & ATATT/A & intergenic & 23 \\
\hline 5 & 130484813 & $\mathrm{~T} / \mathrm{A}$ & intergenic & 23 \\
\hline 6 & 14806096 & GTGACAT/G & intergenic & 23 \\
\hline 6 & 85335098 & $\mathrm{~T} / \mathrm{A}$ & intergenic & 23 \\
\hline 9 & 96708343 & $\mathrm{~A} / \mathrm{G}$ & intergenic & 23 \\
\hline 11 & 36910028 & TAA/T & intergenic & 23 \\
\hline 14 & 25819285 & $\mathrm{CAT} / \mathrm{C}$ & intergenic & 23 \\
\hline 21 & 16468682 & TACAA/T & intergenic & 23 \\
\hline 3 & 64430551 & $\mathrm{G} / \mathrm{C}$ & upstream & 23 \\
\hline 6 & 32083175 & $\mathrm{C} / \mathrm{T}$ & upstream & 23 \\
\hline 10 & 62493816 & $\mathrm{G} / \mathrm{A}$ & upstream & 23 \\
\hline 11 & 93584075 & $\mathrm{~T} / \mathrm{A}$ & upstream & 23 \\
\hline 12 & 114843606 & $\mathrm{C} / \mathrm{T}$ & upstream & 23 \\
\hline 16 & 87638576 & $\mathrm{G} / \mathrm{A}$ & upstream & 23 \\
\hline 19 & 47991316 & TTAAA/T & upstream & 23 \\
\hline 5 & 123647724 & $\mathrm{GACA} / \mathrm{G}$ & downstream & 22.9 \\
\hline 5 & 139749184 & $\mathrm{G} / \mathrm{A}$ & downstream & 22.9 \\
\hline 7 & 27197601 & $\mathrm{C} / \mathrm{G}$ & downstream & 22.9 \\
\hline 8 & 80678427 & $\mathrm{~T} / \mathrm{C}$ & downstream & 22.9 \\
\hline 11 & 61513671 & CAGAG/C & downstream & 22.9 \\
\hline 11 & 125830697 & $\mathrm{~T} / \mathrm{C}$ & downstream & 22.9 \\
\hline 12 & 48396920 & $\mathrm{G} / \mathrm{T}$ & downstream & 22.9 \\
\hline 15 & 100514417 & $\mathrm{C} / \mathrm{T}$ & downstream & 22.9 \\
\hline 16 & 31000726 & TAGAAATGGGACTCTGAGGGCTAAC/T & downstream & 22.9 \\
\hline 1 & 41314741 & $\mathrm{CA} / \mathrm{C}$ & intergenic & 22.9 \\
\hline 1 & 92081030 & $\mathrm{~T} / \mathrm{A}$ & intergenic & 22.9 \\
\hline 1 & 96499830 & $\mathrm{~T} / \mathrm{A}$ & intergenic & 22.9 \\
\hline 2 & 216776870 & $\mathrm{~T} / \mathrm{A}$ & intergenic & 22.9 \\
\hline 3 & 6513635 & CGACA/C & intergenic & 22.9 \\
\hline 3 & 44148568 & ATGCT/A & intergenic & 22.9 \\
\hline 4 & 31809946 & $\mathrm{C} / \mathrm{CACAC}$ & intergenic & 22.9 \\
\hline 5 & 31024929 & $\mathrm{TA} / \mathrm{T}$ & intergenic & 22.9 \\
\hline 6 & 40146909 & ATAAAT/A & intergenic & 22.9 \\
\hline 7 & 9863374 & $\mathrm{TA} / \mathrm{T}$ & intergenic & 22.9 \\
\hline 7 & 27252670 & $\mathrm{~A} / \mathrm{G}$ & intergenic & 22.9 \\
\hline 10 & 86871665 & $\mathrm{TA} / \mathrm{T}$ & intergenic & 22.9 \\
\hline 11 & 42520473 & $\mathrm{~T} / \mathrm{A}$ & intergenic & 22.9 \\
\hline 15 & 95252667 & $\mathrm{~T} / \mathrm{A}$ & intergenic & 22.9 \\
\hline 1 & 40155486 & $\mathrm{~A} / \mathrm{G}$ & upstream & 22.9 \\
\hline 1 & 48965535 & TAAAC/T & upstream & 22.9 \\
\hline 2 & 219722664 & $\mathrm{~T} / \mathrm{C}$ & upstream & 22.9 \\
\hline 5 & 117901084 & $\mathrm{~T} / \mathrm{A}$ & upstream & 22.9 \\
\hline 7 & 20373528 & TTA/T & upstream & 22.9 \\
\hline 7 & 44618599 & $\mathrm{~T} / \mathrm{A}$ & upstream & 22.9 \\
\hline 9 & 107694522 & $\mathrm{C} / \mathrm{T}$ & upstream & 22.9 \\
\hline 11 & 63869107 & $\mathrm{~T} / \mathrm{A}$ & upstream & 22.9 \\
\hline 1 & 3801973 & $\mathrm{~T} / \mathrm{C}$ & downstream & 22.8 \\
\hline 4 & 61659482 & $\mathrm{C} / \mathrm{CCTT}$ & downstream & 22.8 \\
\hline
\end{tabular}


Figure 8: Gene Ontology (GO) terms enriched in the set of 81 genes that harbored the 95 missense variants predicted to be pathogenic. Within the three domains, the top ten terms with significant enrichment are shown in red $(\mathrm{P}<0.05)$, terms with non-significant enrichment are in dark red, and the ancestor terms are shown in black.

\begin{tabular}{|l|l|l|l|l|}
\hline 5 & 123799069 & $\mathrm{C} / \mathrm{G}$ & downstream & 22.8 \\
\hline 5 & 156186841 & $\mathrm{G} / \mathrm{A}$ & downstream & 22.8 \\
\hline 7 & 115147752 & $\mathrm{C} / \mathrm{A}$ & downstream & 22.8 \\
\hline 8 & 28911173 & AGACT/A & downstream & 22.8 \\
\hline 9 & 127642032 & $\mathrm{CAT} / \mathrm{C}$ & downstream & 22.8 \\
\hline 12 & 6054748 & $\mathrm{~T} / \mathrm{C}$ & downstream & 22.8 \\
\hline 12 & 53806714 & $\mathrm{CTG} / \mathrm{C}$ & downstream & 22.8 \\
\hline 14 & 35903276 & GGACATTTA/G & downstream & 22.8 \\
\hline 15 & 92716979 & $\mathrm{~T} / \mathrm{G}$ & downstream & 22.8 \\
\hline 2 & 4155192 & $\mathrm{~T} / \mathrm{A}$ & intergenic & 22.8 \\
\hline 2 & 143562694 & $\mathrm{~T} / \mathrm{G}$ & intergenic & 22.8 \\
\hline 2 & 222173880 & $\mathrm{~T} / \mathrm{A}$ & intergenic & 22.8 \\
\hline 4 & 83012112 & $\mathrm{~T} / \mathrm{A}$ & intergenic & 22.8 \\
\hline 4 & 83119414 & $\mathrm{~T} / \mathrm{A}$ & intergenic & 22.8 \\
\hline 5 & 107795557 & $\mathrm{~T} / \mathrm{A}$ & intergenic & 22.8 \\
\hline 5 & 116888166 & $\mathrm{~T} / \mathrm{A}$ & intergenic & 22.8 \\
\hline 5 & 166025268 & $\mathrm{C} / \mathrm{T}$ & intergenic & 22.8 \\
\hline 7 & 25314445 & T/A & intergenic & 22.8 \\
\hline 10 & 59358896 & T/A & intergenic & 22.8 \\
\hline 9 & 75829027 & T/A & intergenic & 22.8 \\
\hline 9 & 116544406 & T/G & intergenic & 22.8 \\
\hline 11 & 58232952 & T/A & intergenic & 22.8 \\
\hline
\end{tabular}


Table F: Non-frameshifting insertion/deletion variants scored with MutPred-Indel

\begin{tabular}{|c|c|c|c|}
\hline Gene & Variant & Score & $\begin{array}{ll}\text { Affected } & \text { Molecular } \\
\text { Mechanisms } & \end{array}$ \\
\hline NM_000183 & T2_I3insT & 0.78938 & NA \\
\hline NM_147196 & K131Dfs*26 & 0.69025 & NA \\
\hline NM_001085451 & S80_H81insSDRLPRRHSHEDQEFRCRS & 0.70536 & NA \\
\hline NM_000492 & L454Afs*1027 & 0.81268 & NA \\
\hline NM_007343 & E89Nfs*216 & 0.70583 & NA \\
\hline NM_001304441 & V191Afs*254 & 0.68519 & $\begin{array}{l}\text { Mn binding }(\mathrm{p}=0.005) ; \text { PPI } \\
\text { hotspot }(\mathrm{p}=0.006) ; \text { Trans- } \\
\text { membrane region }(\mathrm{p}=0.008) \text {; } \\
\text { PPI residue }(\mathrm{p}=0.011) ; \\
\text { Strand }(\mathrm{p}=0.016)\end{array}$ \\
\hline NM_000423 & S101_F102insG & 0.6849 & NA \\
\hline NM_003771 & S458Rfs*10 & 0.71099 & NA \\
\hline NM_002144 & A2_P28insHSA & 0.77485 & $\mathrm{NA}$ \\
\hline NM_138690 & A1028Gfs*8 & 0.69605 & NA \\
\hline NM_002152 & D261Vfs*439 & 0.68652 & NA \\
\hline NM_002152 & E204_A205insE & 0.68487 & $\mathrm{NA}$ \\
\hline
\end{tabular}


Table G: Splice variants scored with MutPred Splice

\begin{tabular}{|l|l|l|l|l|}
\hline Gene & Variant & Genomic Coordinates & Score & Confident Hypotheses \\
\hline NP_001091994.1 & I229T & chr19,17648350,+ & 0.68 & \\
\hline NP_775815.2 & I229T & chr19,17648350,+ & 0.68 & \\
\hline NP_115702.1 & Q65P & chr4,944210,+ & 0.78 & \\
\hline NP_001727.1 & D2N & chr19,47823038,+ & 0.8 & Loss of natural 3' SS \\
\hline NP_702918.1 & T218T & chr9,131235321,+ & 0.81 & \\
\hline NP_702915.1 & T218T & chr9,131235321,+ & 0.81 & \\
\hline NP_002531.3 & T213T & chr9,131235321,+ & 0.81 & \\
\hline NP_702914.1 & T237T & chr9,131235321,+ & 0.81 & \\
\hline NP_702917.1 & T262T & chr9,131235321,+ & 0.81 & \\
\hline NP_702913.1 & T301T & chr9,131235321,+ & 0.81 & \\
\hline NP_001229281.1 & T232T & chr9,131235321,+ & 0.81 & \\
\hline NP_001229283.1 & T156T & chr9,131235321,+ & 0.81 & \\
\hline NP_702911.1 & T237T & chr9,131235321,+ & 0.81 & \\
\hline NP_001229282.1 & T237T & chr9,131235321,+ & 0.81 & \\
\hline NP_702910.1 & T281T & chr9,131235321,+ & 0.81 & \\
\hline NP_000322.2 & T77S & chr11,18290880,+ & 0.83 & \\
\hline NP_954630.1 & T77S & chr11,18290880,+ & 0.83 & \\
\hline NP_001171477.1 & T77S & chr11,18290880,+ & 0.83 & \\
\hline NP_065946.2 & A357A & chr19,35506729,+ & 0.86 & Cryptic 3' SS \\
\hline NP_001129671.1 & A350A & chr19,35506729,+ & 0.87 & Cryptic 3' SS \\
\hline NP_001018101.1 & Q175Q & chr15,57918090,+ & 0.87 & \\
\hline NP_001018100.1 & Q175Q & chr15,57918090,+ & 0.87 & \\
\hline NP_689664.3 & Q175Q & chr15,57918090,+ & 0.87 & \\
\hline NP_001018110.1 & Q175Q & chr15,57918090,+ & 0.87 & \\
\hline NP_055146.1 & S481S & chr4,139100372,- & 0.88 & \\
\hline NP_001185763.2 & A1757T & chr1,144868170,- & 0.91 & Cryptic 5' SS \\
\hline NP_055459.4 & A1757T & chr1,144868170,- & 0.91 & Cryptic 5' SS \\
\hline NP_001185761.1 & A1651T & chr1,144868170,- & 0.91 & Cryptic 5' SS \\
\hline
\end{tabular}

Table I: Top 5 diseases enriched in the set of 83 genes containing predicted pathogenic missense variants

\begin{tabular}{|l|l|l|l|}
\hline Disease term & $\begin{array}{l}\text { Number } \\
\text { of genes }\end{array}$ & Entrez gene IDs & $\begin{array}{l}\text { Benjamini- } \\
\text { Hochberg ad- } \\
\text { justed } P \text {-value }\end{array}$ \\
\hline Renal diabetes & 5 & $55748,1636,2646,9844,6580$ & 0.0005 \\
\hline Cardiovascular Diseases & 8 & $\begin{array}{l}4153,350,10060,3816,1636,4624, \\
26462153\end{array}$ & 0.0007 \\
\hline Ischemia & 5 & $4153,10060,9620,1636,2153$ & 0.0007 \\
\hline Respiratory Tract Diseases & 7 & $\begin{array}{l}4153,27294,5270,1285,1636,27159 \\
2153\end{array}$ & 0.0007 \\
\hline Lung Diseases & 7 & $4153,27294,5270,1285,1636$ & 0.0007 \\
\hline
\end{tabular}

